\title{
Correspondência entre obesidade e hiperlipidemia em cães
}

\author{
Correspondence between obesity and hyperlipidemia in dogs
}

\section{Márcio Antonio Brunetto ${ }^{\text {I* }}$ Sandra NogueiraII Fabiano César SáIII Mayara Peixoto ${ }^{I}$ Ricardo Souza Vasconcellos $^{\mathrm{I}}$ Antonio José Ferraudo ${ }^{\mathrm{IV}}$ Aulus Cavalieri Carciofi ${ }^{\mathrm{I}}$}

RESUMO

Os efeitos deletérios do excesso de peso sobre a saúde dos cães são bastante citados na literatura, mas pouco investigados. O termo hiperlipidemia refere-se ao aumento da concentração de lipídeos (colesterol, triglicérides ou ambos) séricos. As desordens lipídicas são relativamente comuns nos cães e essas condições podem ocorrer como resultado de um defeito primário no metabolismo de lipoproteínas ou como consequência de doença sistêmica subjacente. O objetivo deste estudo foi avaliar a frequência de animais obesos que apresentam alterações nas concentrações de lipídios séricos $e$ analisar a possível correspondência dessa condição com grau de obesidade, sexo, idade e castração. Foram incluídos 30 cães obesos com escore de condição corporal (ECC) igual ou superior a 8 (em escala de um a nove, com nove representando obesidade acentuada) e um grupo controle de dez animais com ECC igual a cinco. Nos animais com ECC $9(n=21)$, foi encontrada frequência de $52,4 \%(n=11)$ de cães com hipercolesterolemia e 33,3\% ( $n=7)$ com hipertrigliceridemia. Nos animais com EEC $8(n=9)$, foi encontrada frequência de $22,2 \%(n=2)$ de hipercolesterolemia e de $33,3 \%(n=3)$ de hipertrigliceridemia. Essas alterações também apresentaram correspondência com o sexo feminino, faixa etária entre quatro e sete anos e a castração. Todavia, a elevação desses metabólitos foi moderada e não se situou em faixa de reconhecido risco à saúde.

Palavras-chave: canino, colesterol, triglicérides, excesso de peso.

\begin{abstract}
The harmful effects of obesity on dog health are quite mentioned in the literature, but little investigated. The term hyperlipidemia refers to the increase of lipid and/or fat concentration (cholesterol, triglycerides or both) in serum. These disorders are relatively common in dogs and may happen as a result of a primary defect in metabolism of lipoproteins or as a consequence of a subjacent systemic disease. The aim of this study was to evaluate the frequency of obese dogs that present alterations on cholesterol and triglycerides serum concentration, and to study the correlation of these disorders with obesity degree, sex, age and castration. Thirty obese dogs with body condition score (BCS) equal or superior to 8 (on a scale between one to nine, and nine representing severe obesity) and a control group of ten dogs with BCS 5 were included. Animals with BCS 9 ( $n=21$ dogs) presented a frequency of $52.4 \%$ ( $n=11$ dogs) of hypercholesterolemia and $33.3 \%(n=7)$ of hypertriglyceridemia. Dogs with BCS $8(n=9)$ presented a frequency of $22,2 \%(n=2)$ of hypercholesterolemia and $33,3 \% \quad(n=3)$ of hypertriglyceridemia. These alterations also presented correspondence with female sex, age between 4 and 7 years and castration. However, the cholesterol and triglycerides increases were moderate, and not remained within the range of recognized health risk.
\end{abstract}

Key words: canine, cholesterol, triglycerides, over weight .

IDepartamento de Clínica e Cirurgia Veterinária, Faculdade de Ciências Agrárias e Veterinárias (FCAV), Universidade Estadual Paulista (Unesp), Campus de Jaboticabal, Via de Acesso Prof. Paulo Donato Castellane, s/n., 14884-900, Jaboticabal, SP, Brasil. E-mail: brunettovet@yahoo.com.br. *Autor para correspondência.

"Royal Canin do Brasil, São Paulo, SP, Brasil.

"IIPrograma de Pós-graduação em Medicina Veterinária (PPGMV), FCAV, Unesp, Câmpus de Jaboticabal, SP, Brasil.

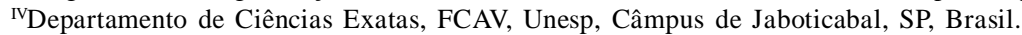




\section{INTRODUÇÃO}

Estudos conduzidos em diferentes países apontam que a incidência de obesidade na população de animais de companhia se situe entre $22 \%$ e $40 \%$. Segundo alguns autores, estima-se que cerca de $34,1 \%$ da população canina americana encontra-se em sobrepeso ou obesa (LUND et al., 2006). Na Austrália, encontrou-se prevalência que variou entre 23 a $41 \%$ dos cães (MCGREEVY et al., 2005). A maioria dos investigadores concorda que, tal como no homem, a incidência de obesidade em animais tende a aumentar. Embora a obesidade seja considerada uma doença essencialmente nutricional, outros fatores que podem predispor o cão ao excesso de peso são a raça, sexo, idade, fatores genéticos, atividade física e densidade energética da dieta (CARCIOFI et al., 2005; GERMAN, 2006; DIEZ \& NGUYEN, 2006). Cães de meia idade a velhos são os mais predispostos e o intervalo de idade de maior prevalência se situa entre 5 a 10 anos (LEWIS et al., 1994; DIEZ \& NGUYEN, 2006; LAFLAMME, 2006). A castração é importante fator de risco para a obesidade em cães, possivelmente devido à diminuição da taxa metabólica basal após a gonadectomia, aumento da ingestão de alimentos e também pelo consequente sedentarismo, sendo as fêmeas castradas mais predispostas do que os machos castrados ao ganho excessivo de peso (GERMAN, 2006; DIEZ \& NGUYEN, 2006). Fatores dietéticos como a alta densidade energética, quantidade de alimento oferecido, número de refeições, fornecimento de petiscos e sobras de mesa também apresentam estreita relação com a gênese da obesidade (GERMAN, 2006). O nutriente que mais eleva o teor energético e a palatabilidade das rações é a gordura, que, por sua vez, é melhor digerida, utilizada e estocada que os carboidratos e proteínas (ROLLS, 2000). Apesar desse fato, a composição nutricional da dieta é menos importante que o consumo energético diário pelo animal, o qual, quando em excesso, independentemente do tipo de alimento, induz ao ganho de peso.

As consequências do excesso de peso sobre a saúde dos cães são bastante citados na literatura, mas pouco investigados. Dentre eles, destacam-se os distúrbios do sistema locomotor (discopatias e ruptura de ligamento cruzado), prejuízos à resposta imunológica, aumento da incidência de endocrinopatias, doenças cardiorespiratórias, afecções reprodutivas, dermatopatias (piodermites e seborréia) e maior incidência de dislipidemias (GERMAN, 2006; LAFLAMME, 2006). O termo hiperlipidemia refere-se ao aumento da concentração de lipídeos (colesterol, triglicérides ou ambos) séricos (ZICKER et al., 2000;
JEUSETTE, et al., 2005; JOHNSON, 2005; SCHENCK, 2006; XENOULIS \& STEINER, 2010). O colesterol e triglicérides são os lipídeos plasmáticos mais relevantes, do ponto de vista clínico, especialmente pela facilidade de sua determinação laboratorial. As desordens lipídicas são relativamente comuns na veterinária, principalmente nos cães. Essas condições podem ocorrer como resultado de defeito primário no metabolismo de lipoproteínas ou como consequência de doença sistêmica subjacente (JOHNSON, 2005; SCHENK, 2006).

Diante do exposto, o objetivo deste estudo foi avaliar a frequência de animais obesos que apresentam alterações nas concentrações de colesterol e triglicérides séricos, bem como analisar a possível correspondência dessa condição com o grau de obesidade, sexo, faixa etária e castração.

\section{MATERIAL E MÉTODOS}

Animais

O experimento incluiu dois grupos de cães, um de trinta animais obesos e outro controle de dez cães com condição corporal ideal. Todos os cães foram provenientes do atendimento de rotina do Serviço de Nutrição Clínica de Cães e Gatos do Hospital Veterinário da FCAV/Unesp. Previamente ao início do estudo, efetuou-se triagem através de exame físico, hematológico, dosagem sérica de alanina amino transferase, fosfatase alcalina, creatinina, uréia, proteína total, albumina, colesterol, triglicerídeos e glicose em jejum. Foram incluídos somente animais obesos por ingestão excessiva de alimento, excluindo-se as demais causas de obesidade.

Diagnóstico da obesidade

O diagnóstico da obesidade foi realizado por meio da inspeção e palpação diretas, com base no escore de condição corporal (ECC) descrito por LAFLAMME (1997). Foram considerados obesos os animais que apresentaram $\mathrm{ECC}=8$, ou seja, costelas não palpáveis debaixo de grande quantidade de gordura subcutânea, depósitos de gordura visíveis na região lombar e na base da cauda, cintura muito pouco aparente ou não visível e curvatura abdominal ausente.

\section{Análises bioquímicas}

Para a realização das avaliações, os animais foram encaminhados ao Laboratório de Pesquisa em Nutrição e Doenças Nutricionais de Cães e Gatos da FCAV/Unesp. Após 12 horas de jejum alimentar, amostras de $5,0 \mathrm{~mL}$ de sangue foram coletadas dos cães por punção da veia jugular e depositadas em tubos de 
vidro para a obtenção de soro. Nas alíquotas de soro, foram analisadas as concentrações de colesterol total e triglicérides. Para essas análises, foram utilizados kits comerciais $^{a}$ seguindo-se as metodologias recomendadas pelos fabricantes. A leitura e obtenção dos resultados foram realizadas em analisador semiautomático ${ }^{\mathrm{b}}$. Todas essas análises foram efetuadas em triplicata.

Análise estatística

Em função da natureza dos dados ser estritamente categórica, empregou-se a análise de correspondência múltipla com o objetivo de explorar as possíveis correspondências entre as variáveis (ou níveis de variáveis) estudadas. As variáveis ou categorias estabelecidas foram: ECC com três níveis (ECC 5, ECC 8, ECC 9); idade dos animais com dois níveis (idade 1, entre 3 e 7 anos; idade 2, acima de 7 anos); colesterolemia com dois níveis (normocolesterolemia, valores entre $154,1 \mathrm{mg} \mathrm{dL}^{-1} \mathrm{e}$ 268,4mg dL $\mathrm{m}^{-1}$; hipercolesterolemia, valores acima de $\left.268,4 \mathrm{mg} \mathrm{dL}^{-1}\right)$; trigliceridemia com dois níveis (normotrigliceridemia, valores entre $16,81 \mathrm{mg} \mathrm{dL}^{-1} \mathrm{e}$ $96,01 \mathrm{mg} \mathrm{dL}^{-1}$; hipertrigliceridemia, valores acima de 96,01 $\mathrm{mg} \mathrm{dL}^{-1}$ ); castrado; não castrado; macho; fêmea. Para esta análise, utilizou-se o software STATISTICAc, segundo metodologia descrita por HAIR et al. (2006). Além disso, as médias de trigliceridemia e colesterolemia dos grupos com ECC 9, 8 e 5 foram comparadas pelo teste de Wilcoxon $(\mathrm{P}<0,05)$.

\section{RESULTADOS E DISCUSSÃO}

No grupo de 30 animais obesos, $22(73,3 \%)$ eram fêmeas e oito machos $(26,7 \%)$. No total, 23 eram não castrados $(76,7 \%)$ e sete $(23,3 \%)$ eram castrados. As raças mais frequentes foram: labrador retriever $(12,9 \%)$, pit bull $(12,9 \%)$, poodle $(9,68 \%)$, beagle $(9,68 \%)$, rottweiller $(9,68 \%)$, cocker $(6,46 \%)$, basset hound
$(3,24 \%)$, dogue alemão $(3,24 \%)$, sendo a maior porcentagem dos cães sem raça definida $(29,03 \%)$. Com relação ao grau de obesidade, 20 animais $(66,7 \%)$ apresentaram escore de condição corporal 9, indicando obesidade pronunciada; $10(33,3 \%)$ dos cães se enquadraram como ECC 8. O grupo controle foi constituído por quatro cães da raça beagle (40\%), dois doberman (20\%), um pastor alemão (10\%), um pequinês (10\%), um pitt bul (10\%) e um pinscher (10\%). Destes, cinco eram fêmeas $(50 \%)$ e cinco eram machos $(50 \%)$. Com relação à condição sexual, nove eram não castrados $(90 \%)$ e um castrado $(10 \%)$. Todos os animais deste grupo apresentaram ECC 5. Os valores de colesterol e triglicérides séricos determinados para os grupos experimentais estão apresentados na tabela 1 .

À análise estatística, foram verificadas diferenças entre a colesterolemia dos cães com obesidade pronunciada (ECC 9) e os demais grupos $(\mathrm{P}<0,05)$, já em relação à trigliceridemia os dois grupos de cães obesos, os cães com ECC8 e ECC 9 apresentaram valores maiores do que os cães em condição ideal $(\mathrm{P}<0,05)$. Os valores de referência adotados para colesterol total e triglicérides foram estipulados considerando-se o método convencional de estabelecimento do intervalo de normalidade descrito por KANEKO et al. (2009). A partir dos valores encontrados para essas duas variáveis no grupo controle, estabeleceu-se como normal a faixa compreendida entre a média mais ou menos dois desvios padrões. Dessa forma, o intervalo de normalidade resultante para a variável colesterolemia compreendeu os valores de 154,1 a $268,4 \mathrm{mg} \mathrm{dL}^{-1}$ e para a variável trigliceridemia, valores de 16,81 a 96,01mg $\mathrm{dL}^{-1}$. Estes foram utilizados para o estabelecimento das categorias na análise estatística de correspondência. Considerando-se os intervalos acima estabelecidos, dos 20 animais com ECC 9, 11 cães apresentaram hipercolesterolemia $(52,4 \%)$ e sete $(33,3 \%)$, hipertrigliceridemia. Nos dez animais com ECC 8, dois

Tabela 1 - Colesterol total e triglicérides séricos dos grupos experimentais obesos e controle (média \pm desvio padrão; mínimo-máximo).

\begin{tabular}{lll}
\hline Grupos & Colesterol total $\left(\mathrm{mg} \mathrm{dL}^{-1}\right)$ & Triglicérides $\left(\mathrm{mg} \mathrm{dL}^{-1}\right)$ \\
\hline Obesos & $332,6 \pm 50,9^{\mathrm{b}}(139,0-368,6)$ & $78,9 \pm 29,9^{\mathrm{a}}(35,1-122,5)$ \\
ECC $8(\mathrm{n}=10)$ & $374,6 \pm 67,9^{\mathrm{a}}(151,7-482,8)$ & $89,3 \pm 40,8^{\mathrm{a}}(43,2-182,4)$ \\
ECC $9(\mathrm{n}=20)$ & \\
Controle & $211,2 \pm 28,6^{\mathrm{b}}(166,9-250,5)$ & \\
ECC $5(\mathrm{n}=10)$ & $56,41 \pm 19,8^{\mathrm{b}}(28,8-81,9)$ \\
\hline
\end{tabular}

a, b - médias na coluna sem uma letra em comum diferem pelo teste de Wilcoxon $(\mathrm{P}<0,05)$. 
apresentaram hipercolesterolemia $(22,2 \%)$ e três $(33,3 \%)$ hipertrigliceridemia.

Embora seja bastante especulado que a obesidade pode alterar as concentrações de colesterol e triglicérides, existem poucas informações referentes à frequência e magnitude desses achados. Estudos já haviam descrito aumento significativo de triglicérides e colesterol plasmático em cães obesos (BARRIE et al., 1993; CHIKAMUNE et al., 1995; BAILHACHE et al., 2003; JEUSETTE et al., 2005). Todavia, deve-se considerar que esses trabalhos, de maneira geral, induziram a obesidade, padronizaram a raça estudada e também a dieta, aspectos que podem influenciar na obtenção dos resultados finais. No presente estudo, o grupo avaliado foi obtido na rotina hospitalar e os cães eram todos domiciliados, não sendo possível dessa forma padronizar esses parâmetros. Por outro lado, os dados aqui encontrados são mais condizentes com a realidade clínica de nosso país.

Alguns autores relataram que o aumento do colesterol total e dos triglicérides plasmáticos resultaram em maior concentração desses metabólitos em todas as frações das lipoproteínas circulantes (CHIKAMUNE et al., 1995) o que não foi possível de ser avaliado no presente estudo, em função da metodologia empregada. No entanto, a mensuração do colesterol total e triglicérides reflete, de forma indireta, o conteúdo sérico das lipoproteínas e fornece informações do estado metabólico das gorduras. Nas situações em que esses metabólitos estão aumentados, subentende-se que uma ou mais lipoproteínas que carreiam esses lipídios vão estar elevadas. Apesar das possíveis limitações dessa inferência, a determinação da concentração sérica de colesterol e triglicérides é ainda a avaliação bioquímica mais utilizada para se determinar anormalidades do metabolismo lipídico na prática clínica (JOHNSON, 2005). Em função disso, essas análises foram escolhidas para a realização do presente levantamento epidemiológico.

A castração é um importante fator de risco para a obesidade em cães, possivelmente devido à diminuição da taxa metabólica basal, aumento do apetite e substituição de massa muscular por tecido adiposo, em função da menor concentração dos hormônios androgênicos. As fêmeas são mais predispostas que os machos ao ganho de peso após a gonadectomia (GERMAN, 2006; DIEZ \& NGUYEN, 2006). Nopresente trabalho, encontrou-se maior frequência de animais obesos não castrados, resultados similares aos encontrados por outros autores nacionais, que descreveram prevalência de $67 \%$ de obesos nessa mesma condição (JERICÓ \& SCHEFFER, 2002), mas diferindo dos resultados encontrados por
ROBERTSON (2003), nos Estados Unidos. Essa discrepância pode ser explicada pelo fato de a técnica de gonadectomia em cães não ser tão difundida em nosso meio como é em países desenvolvidos (JERICÓ \&SCHEFFER, 2002).

O mapa perceptual gerado a partir do emprego da análise de correspondência múltipla, apresentado na figura 1, demonstra que as variáveis obesidade pronunciada (ECC 9), idade acima de 7 anos, fêmeas e castração apresentaram maior correspondência com hipercolesterolemia e hipertrigliceridemia. A análise multivariada de dados tem sido uma ferramenta poderosa, cada vez mais empregada em ensaios clínicos humanos. A correspondência entre as variáveis é verificada pela proximidade geométrica delas no mapa perceptual.

Os possíveis efeitos deletérios da hiperlipidemia crônica sobre a saúde dos cães ainda são desconhecidos. A hipercolesterolemia tem sido associada a lesões oculares e a hipertrigliceridemia pode induzir pancreatite aguda, segundo JEUSETTE et al. (2005), embora esses autores não tenham avaliado e comprovado essa afirmação. Em contraste com humanos, a aterosclerose é rara em cães obesos e isso pode ser explicado em função do metabolismo lipídico da espécie, que se caracteriza por apresentar maiores concentrações de lipoproteínas de alta densidade circulantes, o que os torna mais resistentes ao desenvolvimento de aterosclerose.

Um estudo demonstrou que somente valores de colesterolemia superiores a $750 \mathrm{mg} \mathrm{dL}^{-1}$ predispõe os cães a desenvolverem aterosclerose e animais nesse estado estão 53 vezes mais susceptíveis a desenvolverem Diabetes mellitus e 51 vezes mais predispostos ao hipotireoidismo (HESS et al., 2003). Alguns autores inclusive classificam os valores de colesterol entre $300 \mathrm{mg} \mathrm{dL}^{-1}$ e $500 \mathrm{mg} \mathrm{dL}^{-1}$ como pouco elevados, entre $500 \mathrm{mg} \mathrm{dL}^{-1}$ e $750 \mathrm{mg} \mathrm{dL}^{-1}$ como moderadamente elevados e somente acima de $750 \mathrm{mg}$ $\mathrm{dL}^{-1}$ como gravemente elevados (WHITNEY, 1992). No grupo de cães obesos aqui avaliado, o maior valor de colesterol encontrado foi de $486,5 \mathrm{mg} \mathrm{dL}^{-1}$, o grupo de cães com ECC 9 apresentou valor médio de colesterol de 374,6 $\pm 67,9 \mathrm{mg} \mathrm{dL}^{-1}$, indicando hipercolesterolemia leve. Os achados de hipertriliceridemia foram ainda mais baixos e em menor frequência. Assim, pode-se considerar que a obesidade influi no metabolismo de gorduras, resultando em importante frequência de animais com hipercolesterolemia e hipertrigliceridemia, todavia, a elevação desses metabólitos nos cães não foi intensa e os valores mais elevados não se situaram em faixa de reconhecido risco à saúde. 


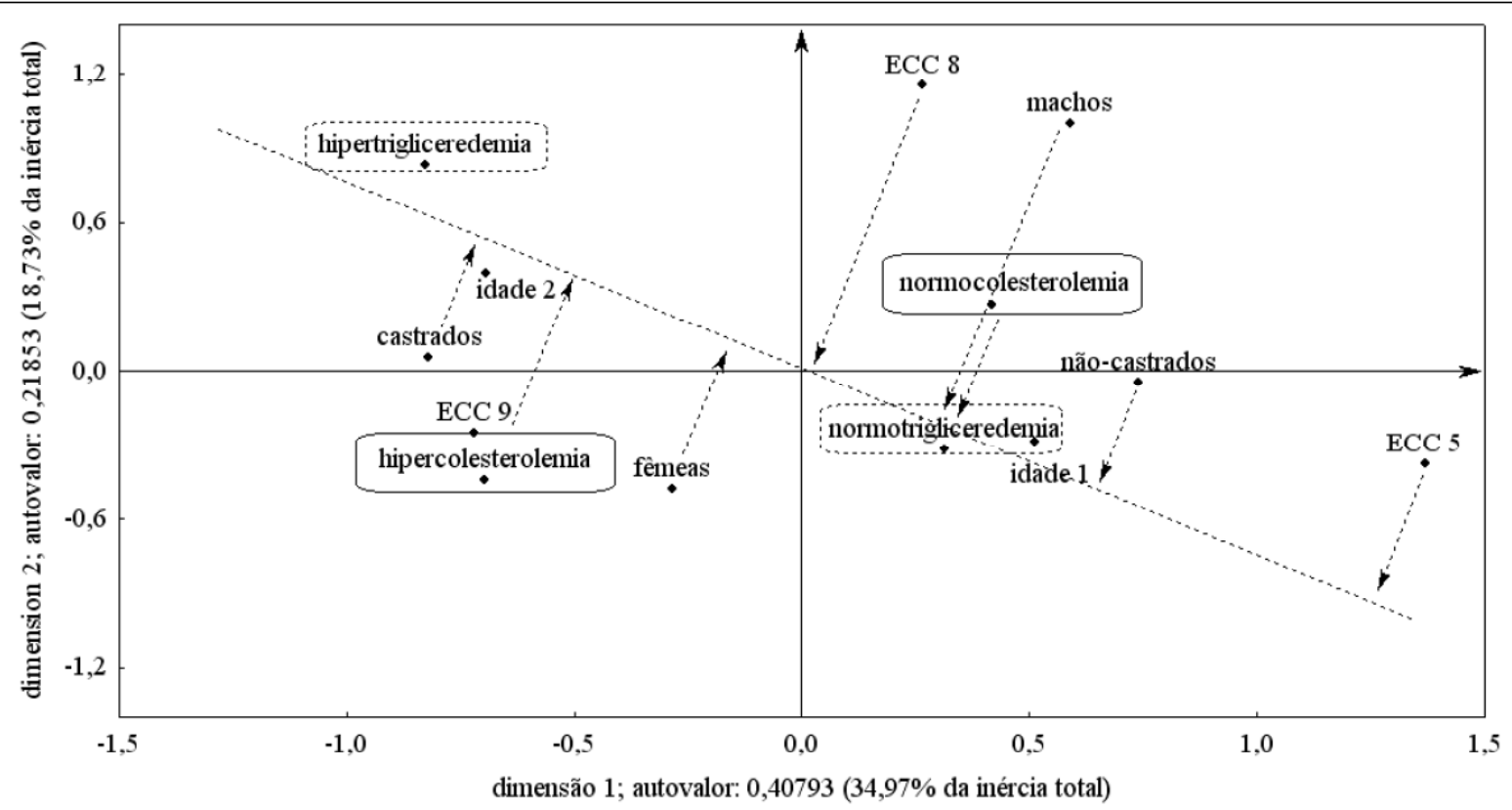

ECC $=$ escore de condição corporal; idade $1=3-7$ anos; idade $2=$ superior a 7 anos; Normocolesterolemia $=154,1-268,4 \mathrm{mg}$ $\mathrm{dL}^{-1}$;Hipercolesterolemia: acima de 268,4mg; Normotrigliceridemia $=16,81-96,01 \mathrm{mgdL}^{-1}$; Hipertrigliceridemia: acima de $96,01 \mathrm{mg}$ $\mathrm{dL}^{-1}$.

Figura 1 - Mapa perceptual gerado pela análise de correspondência multivariada, obtido entre as variáveis colesterolemia, trigliceridemia, grau de obesidade, idade, sexo e castração.

\section{CONCLUSÃO}

Os dados do presente estudo sugerem alta frequência de hipercolesterolemia e hipertrigliceridemia em cães obesos, sendo classificadas como leve. O aumento desses metabólitos apresentou correspondência com o grau de obesidade, sexo feminino, idade acima de sete anos e castração.

\section{AGRADECIMENTOS}

Ao Conselho Nacional de Desenvolvimento Científico e Tecnológico (CNPq), pela concessão de bolsa de doutorado para o primeiro autor. À Fundação de Amparo à Pesquisa do Estado de São Paulo (FAPESP), pela concessão de auxílio financeiro para a execução deste projeto. À MOGIANA ALIMENTOS S.A. (GUABI), pelo suporte financeiro ao Serviço de Nutrição Clínica de Cães e Gatos do Hospital Veterinário da FCAV/Unesp e ao Laboratório de Pesquisa em Nutrição e Doenças Nutricionais de Cães e Gatos do Departamento de Clínica e Cirurgia Veterinária da FCAV/Unesp, Campus de JaboticabalSP.

\section{FONTES DE AQUISIÇÃO}

a - Kit de colesterol e triglicérides, LABTEST, Lagoa Santa MG, Brasil

b - LABTEST, modelo LABQUEST BIO 2000, Lagoa Santa MG, Brasil

c - STATISTICA (Data analysis software system), STATSOFT, Inc. (2004), OK, USA.

\section{REFERÊNCIAS}

BAILHACHE, E. et al. Lipoprotein abnormalities in obese insulinresistant dogs. Metabolism, v.52, p.559-564, 2003. Disponível em: <http://www.sciencedirect.com/science?_ob=ArticleURL\&_udi=B6WN448RWMFF-8\&_user $=972052 \&$ _coverDate $=05 \% 2$ F $31 \% 2$ F 200 $3 \&$ rdoc $=1 \&$ \& mt $=$ high\&_orig =search\&_origi $\mathrm{n}=\mathrm{search} \& \&_{\text {s ort }}=\mathrm{d} \& \&_{-} \mathrm{doc}$ anchor $=\&$ view $=\mathrm{c} \&$ _ searchStrId $=1538518934 \&$ _rerunOrigin $=$ google $\& \_a c c t=C 00$ 0049647 \&_version $=1$ \&_urlVersion $=0 \&$ _userid $=972052$ \&md5=e8dba0768faac17c6917be4902ef83e9\&searchtype $=a>$. Acesso em: 10 nov. 2008. doi: 10.1053/meta.2003.50110.

BARRIE, J. et al. Plasma cholesterol and lipoprotein concentrations in the dog: effect of age, breed, gender and endocrine disease. Journal of Small Animal Practice, v. 34 p.507-512, 1993. Disponível em: <http:// onlinelibrary.wiley.com/doi/10.1111/jsap.1993.34.issue-10/ issuetoc>. Acesso em: 12 nov. 2008. doi: 10.1111/j.17485827.1993.tb03523.x.

BAUER, J.E. Comparative lipid and lipoprotein metabolism. Veterinary Clinical Pathology, v.25, p.49-56, 1996. Disponível em: http://onlinelibrary.wiley.com/doi/10.1111/ j.1939-165X.1996.tb00968.x/abstract. Acesso em: 10 nov. 2010. doi: 10.1111/j.1939-165X.1996.tb00968.x.

CARCIOFI, A.C. et al. A weight loss protocol and owners participation in the treatment of canine obesity. Ciência Rural, v.35, p.1331-1338, 2005. Disponível em: <http:// ww w.s cie 1 o.br/s c i e 1 o.ph p ? p id = S $0103-$ $84782005000600016 \&$ script $=$ sci_arttext $>$. Acesso em: 08 jul. 2009. doi: 10.1590/S0103-84782005000600016. 
CHIKAMUNE, T. et al. Serum lipid and lipoprotein concentrations in obese dogs. Journal of Veterinary Medical Science, v.57, p.595-598, 1995. Disponível em: <http://www.journalarchive.jst.go.jp/ jn lpdf.ph p ? cdjournal=jvms $1991 \&$ cdvol= $57 \&$ noissue $=4 \&$ startpage $=595 \&$ lang $=$ en $\&$ from $=$ jnltoc $>$. Acesso em: 10 jun. 2008. doi: 10.1016/S0167-5877(03)00009-6.

DIEZ, M.; NGUYEN, P. Obesity: epidemiology, pathophysiology and management of the obese dog. In: PIBOT, P. et al. Encyclopedia of canine clinical nutrition. Airmargues: Diffo Print, 2006. p.2-57.

GERMAN, A.J. The growing problem of obesity in dogs and cats. Journal of Nutrition, v.136, p.1940S-1946S, 2006. Disponível em: http://jn.nutrition.org/cgi/reprint/136/7/1940S. Acesso em: 08 nov. 2009

HAIR, J. et al. Multivariate data analysis. 6.ed. Upper Saddle River: Prentice-Hall, 2006. 300p.

HESS, R.S. et al. Association between diabetes mellitus, hypothyroidism or hyperadrenocorticism, and atherosclerosis in dogs. Journal of Veterinary Internal Medicine, v.17, p.489-494, 2003. Disponível em: <http://onlinelibrary.wiley.com/ doi/10.1111/j.1939-1676.2003.tb02469.x/pdf>. Acesso em: 20 jul. 2009. doi: 10.1111/j.1939-1676.2003.tb02469.x.

JERICÓ, M.M.; SCHEFFER, K.C. Epidemiological aspects of obese dogs in the city of Sao Paulo. Clinica Veterinária, v.37, p.25-29, 2002.

JEUSETTE, I.C. et al. Influence of obesity on plasma lipid and lipoprotein concentrations in dogs. American Journal of Veterinary Research, v.66, p.81-86, 2005. Disponível em: <http://www.avma.org/avmacollections/obesity_dogs/ ajvr_66_1_81.pdf>. Acesso em: 11 abr. 2009. doi: 10.2460/ ajvr.2005.66.81.

JOHNSON, M.C. Hyperlipidemia disorders in dogs. Compendium on Continuing Education for the Practicing Veterinarian, v.27, p.361-370, 2005. Disponível em: <https:/ /secure.vlsstore.com/Media/PublicationsArticle/ PV_27_05_361.pdf>. Acesso em: 08 nov. 2008.

KANEKO, J.J. et al. Concepts of normality in clinical biochemistry. In: KANEKO, J.J. Clinical biochemistry of domestic animals. 6.ed. San Diego: Academic, 2009. p.125 .

LAFLAMME, D. Development and validation of a body condition score system for dogs. Canine Practice, v.22, n.4, p.10-15, 1997.

LAFLAMME, D.P. Understanding and managing obesity in dogs and cats. Veterinary Clinics of the North America, v.36, p.1283-1295, 2006. Disponível em: <http:// vetsmall.theclinics.com/issues/contents?issue_key=S01955616\%2806\%29X0029-3>. Acesso em: 12 nov. 2008.
LEWIS, L.D. et al. Obesity. In: Small animal clinical nutrition III. Topeka: Mark Morris Institute, 1994. p.1-39.

LUND, E.M. et al. Prevalence and risk factors for obesity in adult dogs from private US veterinary practices. International Journal of Applied Research in Veterinary Medicine, v.4, p.177-186, 2006. Disponível em: <http://jarvm.com/ articles/Vol4Iss2/Lund.pdf>. Acesso em: 12 nov. 2008.

MCGREEVY, P.D. et al. Prevalence of obesity in dogs examined by Australian Veterinary Practices and the risk factors involved. Veterinary Record, v.156, p.695-702, 2005. Disponível em: http://veterinaryrecord.bmj.com/content/156/22/695.full.pdf. Acesso em: 12 nov. 2008. doi: 10.1136/vr.156.22.695.

ROBERTSON, I.D. The association of exercise, diet and other factors with owner-perceived obesity in privately owned dogs from metropolitan Perth, WA. Preventive Veterinary Medicine, v.58, p.75-83. 2003. Disponível em: <http:// www.sciencedirect.com/science?_ob=ArticleURL\&_udi=B6TBK4808M5M-2\&_user=972052\&_coverDate $=04 \% 2$ F30\%2F2003 \&_rdoc $=1$ \&_fmt=high\&_orig $=$ search\&_origin $=$ search \&_sort $=$ d\&_docanchor $=\& v i e w=c \& \_s e a r c h S t r I d=1538609638 \& \_$rerunO rigin $=$ google $\& \_a c c t=C 000049647 \&$ \&version $=1 \&$ \&urlVersion $=0$ \&_u se r i d $=972052 \& \mathrm{~m} \mathrm{~d} 5=\mathrm{c}$ a f f 2 a 22082 140ce8abfc23661c07b3b\&searchtype $=a>$. Acesso em: 12 nov. 2008. doi: 10.1016/S0167-5877(03)00009-6.

ROLLS, B.J. The role of energy density in the overconsumption of fat. Journal of Nutrition, v.130, p.268S-271S, 2000. Disponível em: <http://jn.nutrition.org/cgi/content/full/130/2/ 268S>. Acesso em: 12 nov. 2008.

SCHENCK, P. Canine hyperlipidemia: causes and nutritional management. In: PIBOT, P. et al. Encyclopedia of canine clinical nutrition. Airmargues: Diffo Print, 2006. p.222250 .

WHITNEY, M.S. Evaluation of hyperlipidemias in dogs and cats. Seminars in Veterinary Medicine and Surgery, v.7, p.292-300, 1992.

XENOULIS, P.G.; STEINER, J.M. Lipid metabolism and hyperlipidemia in dogs. Veterinary Journal, v.183, p.1221, 2010 Disponível em: <http://www.sciencedirect.com/ science?_ob=ArticleURL\&_udi=B6WXN-4VFBYFP$1 \&$ user $=972052 \&$ _coverDate $=01 \% 2$ F3 1\%2F $2010 \&$ _rdoc $=1 \&$ \&mt $=$ high\&_orig $=$ search\&_origin $=$ search\&_sort $=\mathrm{d}$ \&_docanchor $=\& v i e w=c \&$ searchStrId $=1538632804 \&$ _rerunOrigin=google\&_acct=C000049647\&_version=1\&_urlVersion=0\&_u $\mathrm{s}$ e r i d $=972052 \& \mathrm{~m} \mathrm{~d} 5=4 \mathrm{~d} 49$ c 9 f 8912 16437 aecc2143303258a6\&searchtype $=\mathrm{a}>$. Acesso em: 12 nov. 2008. doi:10.1016/j.tvj1.2008.10.011.

ZICKER, S.C. et al. Endocrine and lipid disorders. In: HAND, M.S. et al. Pocket companion to small animal clinical nutrition. Topeka: Mark Morris Institute, 2000. p.762. 